Check for updates

Cite this as: $B M J$ 2021;373:n900 http://dx.doi.org/10.1136/bmj.n900 Published: 13 April 2021

\section{Proposed reforms of the Mental Health Act}

In this editorial by Alison Beck, Paul Farmer, and Til Wykes (BMJ 2021;372;n727; doi:) we incorrectly referred to the UK Mental Health Act. As our correspondents have pointed out, there is no such law as health legislation is a devolved matter. The online version has been amended to reflect this. 